\title{
Particle Spin From Representations of the Diffeomorphism Group
}

\author{
Gerald A. Goldin ${ }^{\star}, 1$ and David H. Sharp ${ }^{2}$ \\ 1 Department of Physics, Princeton University, Princeton, NJ 08544, USA \\ 2 Theoretical Division, Los Alamos National Laboratory, Los Alamos, NM 87545, USA
}

\begin{abstract}
The semidirect product $\mathscr{S} \wedge \mathscr{K}$ of Schwartz' space $\mathscr{S}$ of functions on $\mathbb{R}^{3}$ with the group $\mathscr{K}$ of diffeomorphisms of $\mathbb{R}^{3}$ provides a model for quantum theory based on local currents. Certain unitary representations of $\mathscr{K}$ are induced by representations of $\overline{\mathrm{SL}(3, \mathbb{R})}$. From the local currents in these representations, we construct the generators of local rigid rotations, with respect to which the Hilbert space decomposes into invariant subspaces of fixed spin carrying representations of local SU(2). The physical interpretation of this procedure is discussed.
\end{abstract}

\section{Introduction}

In the local current algebra formulation of non-relativistic quantum theory, systems of spinless particles are described by means of representations of the infinite-dimensional Lie algebra generated by the fixed-time operators $\varrho(f)=\int \varrho(\mathbf{x}) f(\mathbf{x}) d \mathbf{x}$ and $J(\mathbf{g})=\int \mathbf{J}(\mathbf{x}) \cdot \mathbf{g}(\mathbf{x}) d \mathbf{x}$, where $\varrho(\mathbf{x})$ is the mass density, $\mathbf{J}(\mathbf{x})$ is the momentum density, and $f$ and $\mathbf{g}$ are test functions vanishing at infinity. The corresponding infinite-dimensional Lie group is a semidirect product $\mathscr{S} \wedge \mathscr{K}$, where $\mathscr{S}$ is Schwartz' space of test functions under addition, and $\mathscr{K}$ is a group of diffeomorphisms of $\mathbb{R}^{3}$ under composition [1-3]. To describe non-relativistic particles with spin, however, it was thought necessary to introduce at the outset additional operators $\Sigma(\mathbf{h})=\int \Sigma(\mathbf{x}) \cdot \mathbf{h}(\mathbf{x}) d \mathbf{x}$, where $\Sigma(\mathbf{x})$ is the spin density [4]. The Lie group generated by the $\Sigma$-operators is the local SU(2) current group [5-9].

We have seen in earlier work that a wide variety of distinct quantummechanical systems can be described by unitarily inequivalent representations of $\mathscr{S} \wedge \mathscr{K}$, without introducing additional physical quantities. For instance, representations of the diffeomorphism group describe fermions as well as bosons without the need for anticommuting field operators [10]. The question arises, then, whether one can obtain particle spin directly from irreducible repre-

* On sabbatical leave (1982-83) from Department of Mathematical Sciences, Northern Illinois University, DeKalb, IL 60115, USA 
sentations of this group, constructing spin density operators $\Sigma(\mathbf{h})$ from the operators $\varrho(f)$ and $J(\mathbf{g})$ within a representation. In other words, let us suppose we had begun the study of quantum theory by investigating the irreducible unitary representations of $\mathscr{S} \wedge \mathscr{K}$ and the correspondence in a representation of the selfadjoint generators to physical measurements. Could we have inferred the possibility of particles with spin, both integral and half-integral?

The purpose of the present paper is to suggest an affirmative answer to this question. We shall interpret subspaces in certain representations of $\mathscr{S} \wedge \mathscr{K}$ as describing particles with integer or half-integer spin. We then construct explicitly a set of operators, the generators of "local rigid rotations," corresponding to the measurement of local angular momentum, which leave the fixed-spin subspaces invariant. These, together with operators corresponding to measurements of local mass density, we call "local measurement operators." We then demonstrate how $\Sigma(\mathbf{h})$ can be obtained in terms of them: the local measurement operators, together with the total momentum, form a complete set of observables in each fixed-spin subspace, and permit the recovery in each subspace of a new representation of $\mathscr{S} \wedge \mathscr{K}$ together with local $\mathrm{SU}(2)$. Thus, the initial representation of $\varrho(f)$ and $J(\mathbf{g})$ alone is sufficient to describe spin degrees of freedom as well as position and momentum degrees of freedom.

In Sect. II we introduce needed notation and review the use of representations of $\overline{\mathrm{SL}(3, \mathbb{R})}$ to induce representations of the diffeomorphism group [11-13]. In Sect. III we interpret the local currents in these representations, constructing the local measurement operators by taking appropriate limits in a representation of $\mathscr{S} \wedge \mathscr{K}$. With respect to local rigid rotations the Hilbert space decomposes into invariant subspaces of fixed spin, within which representations of local SU(2) are constructed. We also remark on the generalization of these results to the case of $N$ particles. In Sect. IV we discuss the physical interpretation of the procedure we have adopted.

Taken together with earlier work [14-15], this paper shows how unitarily inequivalent representations of the same group $\mathscr{S} \wedge \mathscr{K}$ can describe many different physical systems, including: systems of $N$ identical particles satisfying Bose or Fermi statistics, particles in two-dimensional space with unusual statistics [16], systems of distinct species of particles having different masses or charges, systems of infinitely many particles in the thermodynamic limit, systems of point dipoles, quadrupoles, etc. [17], and now particles with integer or half-integer spin. Such universality is possible owing to the infinitely many degrees of freedom embodied in the group of diffeomorphisms.

\section{Representations of the Diffeomorphism Group Induced from $\overline{\operatorname{SL}(3, \mathbb{R})}$}

The operators $\varrho(f)$ and $J(\mathbf{g})$ satisfy the infinite-dimensional Lie algebra (in units where $\hbar=1$ )

$$
\begin{aligned}
{\left[\varrho\left(f_{1}\right), \varrho\left(f_{2}\right)\right] } & =0, \\
{[\varrho(f), J(\mathbf{g})] } & =i \varrho(\mathbf{g} \cdot \nabla f), \\
{\left[J\left(\mathbf{g}_{1}\right), J\left(\mathbf{g}_{2}\right)\right] } & =i J\left(\left[\mathbf{g}_{1}, \mathbf{g}_{2}\right]\right),
\end{aligned}
$$


where $\left[\mathbf{g}_{1}, \mathbf{g}_{2}\right]=\mathbf{g}_{2} \cdot \nabla \mathbf{g}_{1}-\mathbf{g}_{1} \cdot \nabla \mathbf{g}_{2}$ is the Lie bracket of the vector fields $\mathbf{g}_{1}$ and $\mathbf{g}_{2}$. The product law for the corresponding Lie group $\mathscr{S} \wedge \mathscr{K}$ is

$$
\left(f_{1}, \boldsymbol{\varphi}_{1}\right)\left(f_{2}, \boldsymbol{\varphi}_{2}\right)=\left(f_{1}+\boldsymbol{\varphi}_{1} f_{2}, \boldsymbol{\varphi}_{1} \boldsymbol{\varphi}_{2}\right),
$$

where $f_{1}$ and $f_{2}$ are Schwartz' space functions, $\boldsymbol{\varphi}_{1}$ and $\boldsymbol{\varphi}_{2}$ are diffeomorphisms of $\mathbb{R}^{3},(\varphi f)(\mathbf{x})=f(\varphi(\mathbf{x}))$, and $\left(\boldsymbol{\varphi}_{1} \boldsymbol{\varphi}_{2}\right)(\mathbf{x})=\boldsymbol{\varphi}_{2}\left(\boldsymbol{\varphi}_{1}(\mathbf{x})\right)$. A continuous unitary representation of $\mathscr{S} \wedge \mathscr{K}$ will be written $\mathscr{U}(f) \mathscr{V}(\boldsymbol{\varphi})$. For $\boldsymbol{\varphi} \in \mathscr{K}$, we denote by $\mathscr{J}_{\boldsymbol{\varphi}}(\mathbf{x})$ the Jacobian matrix of $\boldsymbol{\varphi}$ at $\mathbf{x}$; i.e. $\left[\mathscr{J}_{\boldsymbol{\varphi}}(\mathbf{x})\right]_{k}^{j}=\partial_{k} \varphi^{j}(\mathbf{x})$.

Let $\boldsymbol{\varphi}_{s}^{\mathbf{g}}: \mathbb{R}^{3} \rightarrow \mathbb{R}^{3}$ for $s \in \mathbb{R}$ be a one-parameter group of diffeomorphisms (a flow) satisfying $\partial_{s} \boldsymbol{\varphi}_{s}^{\mathbf{g}}=\mathbf{g} \circ \boldsymbol{\varphi}_{s}^{\mathbf{g}}$, with $\boldsymbol{\varphi}_{s=0}^{\mathbf{g}}(\mathbf{x})=\mathbf{x}$. Then we have $\exp [i s J(\mathbf{g})]$ $=\mathscr{V}\left(\boldsymbol{\varphi}_{s}^{\mathbf{g}}\right)$. Since $\mathbf{g}$ and all of its derivatives vanish rapidly at infinity, $\boldsymbol{\varphi}_{s}^{\mathbf{g}}(\mathbf{x})$ rapidly approaches $\mathbf{x}$ when $|\mathbf{x}|$ becomes large (for fixed s), and its Jacobian matrix $\left[\mathscr{J}_{\varphi_{s}^{g}}(\mathbf{x})\right]_{k}^{j}$ approaches the identity matrix $\delta_{k}^{j}$. We will take $\mathscr{K}$ to be the closure of the group generated by all such one-parameter groups of diffeomorphisms, with respect to a topology of uniform convergence in all derivatives. Thus elements of $\mathscr{K}$ satisfy these conditions at infinity.

For fixed $\mathbf{x}$, define the mapping $h_{\mathbf{x}}: \mathscr{K} \rightarrow \operatorname{SL}(3, \mathbb{R})$ by $h_{\mathbf{x}}(\boldsymbol{\varphi})=\left(\operatorname{det} \mathscr{J}_{\boldsymbol{\varphi}}(\mathbf{x})\right)^{-1 / 3}$ - $\mathscr{I}_{\boldsymbol{\varphi}}(\mathbf{x})$. Let the stability group (or "little group") be given by $\mathscr{K}_{\mathbf{x}}=\{\boldsymbol{\varphi} \in \mathscr{K}: \boldsymbol{\varphi}(\mathbf{x})=\mathbf{x}\}$; then the restriction $h_{\mathbf{x}}: \mathscr{K}_{\mathbf{x}} \rightarrow \operatorname{SL}(3, \mathbb{R})$ is a continuous homomorphism. If $\mathbf{x}_{t}$ (for $0<t \leqq 1$ ) is a continuous path in $\mathbb{R}^{3}$ from infinity to $\mathbf{x}$, then $h_{\mathbf{x}_{t}}(\boldsymbol{\varphi})$ defines a continuous path (for $0 \leqq t \leqq 1$ ) from the identity to $h_{\mathbf{x}}(\boldsymbol{\varphi})$ in $\operatorname{SL}(3, \mathbb{R})$. Such a path corresponds to an element of the universal covering group $\overline{\mathrm{SL}}(3, \mathbb{R})$. Any alternative path $\mathbf{x}_{t}^{\prime}$ from infinity to $\mathbf{x}$ may be deformed continuously into $\mathbf{x}_{t}$; their images $h_{\mathbf{x}_{t}}(\boldsymbol{\varphi})$ and $h_{\mathbf{x}_{t}}(\boldsymbol{\varphi})$ in $\operatorname{SL}(3, \mathbb{R})$ are thus seen to be homotopic. Hence we have a well-defined map $\tilde{h}_{\mathbf{x}}: \mathscr{K} \rightarrow \overline{\operatorname{SL}(3, \mathbb{R})}$. It is straightforward to verify that

$$
\tilde{h}_{\mathbf{x}}\left(\boldsymbol{\varphi}_{1} \boldsymbol{\varphi}_{2}\right)=\tilde{h}_{\mathbf{x}}\left(\boldsymbol{\varphi}_{1}\right) \tilde{h}_{\boldsymbol{\varphi}_{1}(\mathbf{x})}\left(\boldsymbol{\varphi}_{2}\right),
$$

whence $\tilde{h}_{\mathbf{x}}$ restricted to $\mathscr{K}_{\mathbf{x}}$ defines a homomorphism from $\mathscr{K}_{\mathbf{x}}$ to $\overline{\operatorname{SL}(3, \mathbb{R})}[13]$.

Next consider a continuous unitary representation $\pi$ of $\overline{\operatorname{SL}(3, \mathbb{R})}$ in a Hilbert space $\mathscr{M}$. This induces a representation $\mathscr{V}$ of $\mathscr{K}$ in the Hilbert space $\mathscr{H}=L^{2}\left(\mathbb{R}^{3}, \mathscr{M}\right)$, given by

$$
[\mathscr{V}(\boldsymbol{\varphi}) \Psi](\mathbf{x})=\pi\left(\tilde{h}_{\mathbf{x}}(\boldsymbol{\varphi})\right) \Psi(\boldsymbol{\varphi}(\mathbf{x}))\left(\operatorname{det} \mathscr{J}_{\boldsymbol{\varphi}}(\mathbf{x})\right)^{1 / 2} .
$$

Schwartz' space is represented in $L^{2}\left(\mathbb{R}^{3}, \mathscr{M}\right)$ by operators $\mathscr{U}(f)$ satisfying

$$
[\mathscr{U}(f) \Psi](\mathbf{x})=\exp [i f(\mathbf{x})] \Psi(\mathbf{x}),
$$

and Eqs. (4) and (5) define a continuous unitary representation of $\mathscr{S} \wedge \mathscr{K}$.

To write the self-adjoint generators in such a representation, let $J_{0}(\mathbf{g})$ $=(2 i)^{-1}(\mathbf{g} \cdot \nabla+\nabla \cdot \mathbf{g})$ acting on the spatial coordinate $\mathbf{x}$. Following the notation of Sijacki [18], let $\Sigma_{0}$ and $\Sigma_{ \pm}$be the operators acting in $\mathscr{M}$ which represent the usual generators of $\mathrm{SU}(2)$, the maximal compact subgroup of $\overline{\mathrm{SL}(3, \mathbb{R})}$, with $\Sigma_{3}=\Sigma_{0}$ and $\Sigma_{ \pm}=\Sigma_{1} \pm i \Sigma_{2}$. Let $T_{\mu}$ for $\mu=-2,-1,0,1,2$ be the operators in $\mathscr{M}$ representing the quadrupole generators of $\overline{\mathrm{SL}(3, \mathbb{R})}$, obeying $T_{-\mu}^{*}=(-1)^{\mu} T_{\mu}$. The representation $\pi$ determines $\Sigma_{0}, \Sigma_{ \pm}$, and $T_{\mu}$. The commutation relations among these generators 
are:

$$
\begin{aligned}
{\left[\Sigma_{0}, \Sigma_{ \pm}\right] } & = \pm \Sigma_{ \pm}, \\
{\left[\Sigma_{+}, \Sigma_{-}\right] } & =2 \Sigma_{0}, \\
{\left[\Sigma_{0}, T_{\mu}\right] } & =\mu T_{\mu} \\
{\left[\Sigma_{ \pm}, T_{\mu}\right] } & =(6-\mu(\mu \pm 1))^{1 / 2} T_{\mu \pm 1}, \\
{\left[T_{2}, T_{-2}\right] } & =-4 \Sigma_{0},
\end{aligned}
$$

with other commutators following from the Jacobi identity.

Define

$$
\begin{aligned}
& G_{0}(\mathbf{g})=\sqrt{\frac{1}{6}}\left(\partial_{1} g^{1}+\partial_{2} g^{2}-2 \partial_{3} g^{3}\right) \\
& G_{1}(\mathbf{g})=-\overline{G_{-1}(\mathbf{g})}=-\frac{1}{2}\left[\left(\partial_{1} g^{3}+\partial_{3} g^{1}\right)+i\left(\partial_{2} g^{3}+\partial_{3} g^{2}\right)\right] \\
& G_{2}(\mathbf{g})=\overline{G_{-2}(\mathbf{g})}=-\frac{1}{2}\left[\left(\partial_{1} g^{1}-\partial_{2} g^{2}\right)+i\left(\partial_{1} g^{2}-\partial_{2} g^{1}\right)\right]
\end{aligned}
$$

Now the representation

$$
\begin{aligned}
& \varrho(f)=f(\mathbf{x}) \cdot I \\
& J(\mathbf{g})=J_{0}(\mathbf{g}) \cdot I+\frac{1}{2}(\operatorname{curl} \mathbf{g}) \cdot \mathbf{\Sigma}+\frac{1}{2} \sum_{\mu=-2}^{2} G_{-\mu}(\mathbf{g}) T_{\mu}
\end{aligned}
$$

gives the self-adjoint generators obtained from Eqs. (4) and (5). One can verify from Eqs. (6)-(8) that the local current algebra of Eq. (1) is satisfied.

The representations of $\overline{\mathrm{SL}(3, \mathbb{R})}$ described by Sijacki can be written as sums of finite-dimensional representations of $\mathrm{SU}(2)$ with various multiplicities, where the operators $T_{\mu}$ intertwine these representations. For example, if $(2 S+1)$ denotes the dimensionality of a "spin $S$ " representation of $\operatorname{SU}(2)$, there exist $\overline{\operatorname{SL}(3, \mathbb{R})}$ representations with $S$-values corresponding to $\left\{0,2^{2}, 3,4^{3}, 5^{2}, 6^{4}, \ldots\right\}$, or $\left\{1,2,3^{2}, 4^{2}\right.$, $\left.5^{3}, 6^{3}, \ldots\right\}$, or $\left\{\frac{1}{2},\left(\frac{3}{2}\right)^{2},\left(\frac{5}{2}\right)^{3},\left(\frac{7}{2}\right)^{4}, \ldots\right\}$, where the exponents indicate multiplicities. There also exist multiplicity-free $\Delta S=2$ representations $\{0,2,4, \ldots\},\{1,3,5, \ldots\}$, and $\left\{\frac{1}{2}, \frac{5}{2}, \frac{9}{2}, \ldots\right\}$.

Since $\overline{\mathrm{SL}(3, \mathbb{R})}$ is non-compact, it has no non-trivial finite-dimensional irreducible representations. Thus if $J(\mathbf{g})$ in Eq. (8) corresponds to a physical measurement for all $\mathbf{g}$, it must for at least some $\mathbf{g}$ describe an experiment in which the particle spin can change. Such a situation could conveivably occur for supermultiplets of hadrons lying on Regge trajectories, for excited states of nuclei [19], or in the presence of a strong non-uniform gravitational field (see discussion below). Alternatively, to describe the usual nonrelativistic physics of particles with fixed spin, we can construct from the $\varrho(f)$ and $J(\mathbf{g})$ in the foregoing representations a class of operators which preserve the SU(2)-invariant subspaces. We do this in the next section. As explained below, plausible physical arguments can be given to suggest that these are the operators corresponding to realizable physical experiments. 


\section{Local Rigid Rotations and their Generators}

Considering the terms in Eq. (8), we propose to interpret the first term $J_{0}(\mathbf{g}) \cdot I$, which acts only on spatial coordinates, as the ordinary (orbital, or kinetic) momentum density averaged with the function $\mathbf{g}$. The second term $(1 / 2)(\operatorname{curl} \mathbf{g}) \cdot \boldsymbol{\Sigma}$ we interpret as the spin density averaged with $(1 / 2)(\operatorname{curl} \mathbf{g})$. Subspaces of $L^{2}\left(\mathbb{R}^{3}, \mathscr{M}\right)$ having a given spin are invariant under these operators. However, the terms $(1 / 2) \sum G_{-\mu}(\mathbf{g}) T_{\mu}$ connect the different spin subspaces. We think of these as infinitesimal generators of non-uniform spatial dilations. One cannot simply set these $T_{\mu}$ terms equal to zero; for while $J_{0}(\mathbf{g}) \cdot I$ and $J(\mathbf{g})$ separately satisfy the third commutation relation of Eq. (1), the combination $J_{0}(\mathbf{g}) \cdot I+(1 / 2)($ curlg $) \cdot \boldsymbol{\Sigma}$ does not.

Suppose then that we wish to measure the $z$-component of total angular momentum (orbital plus spin) in a bounded region with cylindrical symmetry about the $z$-axis. For specificity, we choose a sphere of radius $R$ centered at the origin, and proceed as follows to construct the appropriate self-adjoint operator. Let $\psi_{s}: \mathbb{R}^{3} \rightarrow \mathbb{R}^{3}$ be the one-parameter group of local rotations given by $\boldsymbol{\psi}_{s}(\mathbf{x})=\mathbf{x}_{s}$, where $\mathbf{x}=\left(x^{1}, x^{2}, x^{3}\right)$, with:

$$
\left.\begin{array}{l}
x_{s}^{1}=(\cos s) x^{1}-(\sin s) x^{2}, \\
x_{s}^{2}=(\sin s) x^{1}+(\cos s) x^{2}, \\
x_{s}^{3}=x^{3}, \\
\mathbf{x}_{s}=\mathbf{x} \text { for }|\mathbf{x}|>R .
\end{array}\right\}|\mathbf{x}| \leqq R
$$

The local rotation $\psi_{s}$ is discontinuous at $|\mathbf{x}|=R$. Now a one-parameter unitary group $V\left(\psi_{s}\right)$ acting in $L^{2}\left(\mathbb{R}^{3}\right)$ is defined by $\left(V\left(\psi_{s}\right) \Phi\right)(\mathbf{x})=\Phi\left(\psi_{s}(\mathbf{x})\right)$. The (discontinuous) vector field $\mathbf{q}$ generating such a rotation is given by

$$
\begin{aligned}
\mathbf{q}(\mathbf{x}) & =\left(-x^{2}, x^{1}, 0\right), & & |\mathbf{x}| \leqq R, \\
& =0, & & |\mathbf{x}|>R .
\end{aligned}
$$

The operator $J_{0}(\mathbf{q})$, defined to be the infinitesimal generator of $V\left(\psi_{s}\right)$, is thus selfadjoint. Let $\chi_{R}(\mathbf{x})=1$ for $|\mathbf{x}| \leqq R$ and $\chi_{R}(\mathbf{x})=0$ for $|\mathbf{x}|>R$. We write formally $J_{0}(\mathbf{q})$ $=(2 i)^{-1}(\mathbf{q} \cdot \nabla+\nabla \cdot \mathbf{q})$, and observe that $J_{0}(\mathbf{q})=J_{0}\left(\left(\chi_{R}(\mathbf{x}) \hat{z}\right) \times \mathbf{x}\right)=\int\left(\mathbf{x} \times \mathbf{J}_{0}(\mathbf{x})\right)$ $\cdot\left(\chi_{R}(\mathbf{x}) \hat{z}\right) d \mathbf{x}$, where $\hat{z}$ denotes the unit vector in the $x^{3}$-direction. Thus $J_{0}(\mathbf{q})$, expressed in units of angular momentum, plausibly corresponds to measurement of the $z$-component of orbital angular momentum in the region $|\mathbf{x}| \leqq R$. The selfadjointness of $J_{0}(\mathbf{q})$ is an interesting property of operators corresponding to measurements of angular momentum in bounded, cylindrically symmetric regions. Its analogue is false if one attempts to write operators for measurements of linear momentum in bounded regions. That is, if $\chi$ is the characteristic function of any bounded region, $J_{0}(\chi \hat{z})=\int \mathbf{J}_{0}(\mathbf{x}) \cdot(\chi(\mathbf{x}) \hat{z}) d \mathbf{x}$ cannot be self-adjoint, since $\chi \hat{z}$ does not generate a transformation group of the region. In what follows, we exploit the selfadjointness of $J_{0}(\mathbf{q})$, where $\mathbf{q}$ generates a local rigid rotation, to construct operators which preserve the SU(2)-invariant subspaces in the representation of Eq. (8). 
Theorem. Let $\mathbf{q}$ be given by Eq. (10), and $\chi_{R}$ be the characteristic function of the region $|\mathbf{x}| \leqq R$. Let $\mathbf{q}_{\alpha}, \alpha=1,2,3, \ldots$, be a sequence of $C^{\infty}$ vector fields with components in Schwartz' space, with $\lim _{\alpha \rightarrow \infty} \mathbf{q}_{\alpha}=\mathbf{q}$ in the sense of a weak limit of distributions; that is, for any $\mathbf{g}$ with components in $\mathscr{S}, \lim _{\alpha \rightarrow \infty} \int \mathbf{q}_{\alpha}(\mathbf{x}) \cdot \mathbf{g}(\mathbf{x}) d \mathbf{x}=\int \mathbf{q}(\mathbf{x})$ - $\mathbf{g}(\mathbf{x})$ d) $\mathbf{x}$. Let $\left\{\Omega_{n}\right\}$ be an orthonormal basis for the representation space $\mathscr{M}$ of $\mathrm{SL}(3, \mathbb{R})$, where $n$ stands for the triple of quantum numbers $(S, M, K) ;(S, M)$ are the $\mathrm{SU}(2)$ quantum numbers corresponding to total spin and the z-component of spin respectively, and $K$ indexes the distinct $\mathrm{SU}(2)$-invariant subspaces having the same value of $S$. Let $D \subseteq L^{2}\left(\mathbb{R}^{3}, \mathscr{M}\right)$ be the domain of finite linear combinations of vectors of the form $\Phi_{n}(\mathbf{x}) \Omega_{n}$, where $\Phi_{n} \in \mathscr{S}$, and let $D_{0}$ be the subspace of $D$ consisting of vectors $\Psi$ for which $\lim _{\alpha \rightarrow \infty} J\left(\mathbf{q}_{\alpha}\right) \Psi$ exists weakly with respect to $D$, where $J\left(\mathbf{q}_{\alpha}\right)$ is given by Eq. (8). That is, $\Psi \in D_{0}$ if and only if there is a vector $\Lambda$ in $L^{2}\left(\mathbb{R}^{3}, \mathscr{M}\right)$ such that for all $\Psi^{\prime} \in D, \quad \lim _{\alpha \rightarrow \infty}\left(\Psi^{\prime}, J\left(\mathbf{q}_{\alpha}\right) \Psi\right)=\left(\Psi^{\prime}, \Lambda\right)$. Then for $\Psi \in D_{0}, \quad \lim _{\alpha \rightarrow \infty} J\left(\mathbf{q}_{\alpha}\right) \Psi$ $=\left(J_{0}(\mathbf{q}) \cdot I+\mathbf{v} \cdot \mathbf{\Sigma}\right) \Psi$ weakly with respect to $D$, where $\mathbf{v}$ is the (constant) characteristic vector field $\mathbf{v}(\mathbf{x})=\chi_{R}(\mathbf{x}) \hat{z}$.

Proof. The domain $D$ is a common dense invariant domain for all the operators $\varrho(f)$ and $J(\mathbf{g})$. Consider the matrix element $\left(\Phi_{n}^{\prime} \Omega_{n}, J\left(\mathbf{q}_{\alpha}\right) \Phi_{m} \Omega_{m}\right)$ as $\alpha \rightarrow \infty$, and look at each term given by Eq. (8). First, we have

$$
\begin{aligned}
& \lim _{\alpha \rightarrow \infty}\left(\Phi_{n}^{\prime} \Omega_{n}, J_{0}\left(\mathbf{q}_{\alpha}\right) \cdot I \Phi_{m} \Omega_{m}\right)=\lim _{\alpha \rightarrow \infty} \delta_{m n} \int \overline{\Phi_{n}^{\prime}(\mathbf{x})} \frac{1}{2 i}\left[\mathbf{q}_{\alpha}(\mathbf{x}) \cdot \nabla \Phi_{m}(\mathbf{x})+\nabla \cdot\left(\mathbf{q}_{\alpha}(\mathbf{x}) \Phi_{m}(\mathbf{x})\right)\right] d \mathbf{x} \\
& =\lim _{\alpha \rightarrow \infty} \delta_{m n} \frac{1}{i} \int \mathbf{q}_{\alpha}(\mathbf{x}) \cdot\left[\overline{\Phi_{n}^{\prime}(\mathbf{x})} \cdot \nabla \Phi_{m}(\mathbf{x})\right] d \mathbf{x}+\lim _{\alpha \rightarrow \infty} \delta_{m n} \frac{1}{2 i} \int\left(\operatorname{div} \mathbf{q}_{\alpha}(\mathbf{x})\right)\left[\overline{\Phi_{n}^{\prime}(\mathbf{x})} \Phi_{m}(\mathbf{x})\right] d \mathbf{x} .
\end{aligned}
$$

The first term of Eq. (11) converges to $\delta_{m n} i^{-1} \int \mathbf{q}(\mathbf{x}) \cdot\left[\overline{\Phi_{n}^{\prime}(\mathbf{x})} \nabla \Phi_{m}(\mathbf{x})\right] d \mathbf{x}$ by the weak convergence of $\mathbf{q}_{\alpha}$ to $\mathbf{q}$. But this convergence also implies the weak convergence of the distribution $\operatorname{div} \mathbf{q}_{\alpha}$ to the distribution divq. As distributions, $\partial_{1} q^{1}(\mathbf{x})$ $=\left(x^{1} x^{2} / R\right) \delta(|\mathbf{x}|-R)$ and $\partial_{2} q^{2}(\mathbf{x})=-\left(x^{1} x^{2} / R\right) \delta(|\mathbf{x}|-R)$, so $\operatorname{div} \mathbf{q}=0$. Thus for any $\Psi, \Psi^{\prime} \in D$, we may write $\lim _{\alpha \rightarrow \infty}\left(\Psi^{\prime}, J_{0}\left(\mathbf{q}_{\alpha}\right) \cdot I \Psi\right)=\left(\Psi^{\prime}, J_{0}(\mathbf{q}) \cdot I \Psi\right)$, where $J_{0}(\mathbf{q})$ is the local orbital angular momentum operator discussed above, acting on the spatial coordinate $\mathbf{x}$.

Next, we have for the second term of Eq. (8),

$\lim _{\alpha \rightarrow \infty}\left(\Phi_{n}^{\prime} \Omega_{n} \frac{1}{2}\left(\operatorname{curl} \mathbf{q}_{\alpha}\right) \cdot \mathbf{\Sigma} \Phi_{m} \Omega_{m}\right)=\lim _{\alpha \rightarrow \infty}\left[\frac{1}{2} \int \overline{\Phi_{n}^{\prime}(\mathbf{x})} \Phi_{m}(\mathbf{x})\left(\operatorname{curl} \mathbf{q}_{\alpha}(\mathbf{x})\right) d \mathbf{x}\right] \cdot\left(\Omega_{n}, \mathbf{\Sigma} \Omega_{m}\right)$.

Of course if $n=(S, M, K)$ and $m=\left(S^{\prime}, M^{\prime}, K^{\prime}\right)$, the inner product $\left(\Omega_{n}, \Sigma \Omega_{m}\right)$ vanishes unless $S=S^{\prime}$ and $K=K^{\prime}$. Now $\mathbf{q}_{\alpha} \rightarrow \mathbf{q}$ implies curl $\mathbf{q}_{\alpha} \rightarrow$ curlq as distributions, and $\left.\operatorname{curl} \mathbf{q}=\hat{z}\left(\partial_{1} q^{2}-\partial_{2} q^{1}\right)=\hat{z}\left[2 \chi_{R}(\mathbf{x})-\left(R^{2}-\left(x^{3}\right)^{2}\right) / R\right) \delta(|\mathbf{x}|-R)\right]$. Thus, in evaluating Eq. (12), we have the desired term $\left(\Phi_{n}^{\prime} \Omega_{n},\left(\chi_{R} \hat{z}\right) \cdot \Sigma \Phi_{m} \Omega_{m}\right)$, and a surface term which equals

$$
-(R / 2) \hat{z} \cdot\left(\Omega_{n}, \Sigma \Omega_{m}\right) \iint_{|\mathbf{x}|=R} \overline{\Phi_{n}^{\prime}(\mathbf{x})} \Phi_{m}(\mathbf{x}) \sin ^{2} \theta d A,
$$

where $\theta$ is the polar angle of $\mathbf{x}$ with $\hat{z}$. The surface term must vanish in order that the result be the inner product of $\Phi_{n}^{\prime} \Omega_{n}$ with a vector in $L^{2}\left(\mathbb{R}^{3}, \mathscr{M}\right)$. It does so for all $\Phi_{n}^{\prime} \Omega_{n} \in D$ if and only if we restrict $\Phi_{m}$ to be a function which vanishes on the 
boundary $|\mathbf{x}|=R$. Note that the finite linear combinations of $\Phi_{m} \Omega_{m}$ under this restriction still form a dense domain in $L^{2}\left(\mathbb{R}^{3}, \mathscr{M}\right)$, which we shall see is the domain $D_{0}$ in the statement of the theorem.

Finally, we consider the third term of Eq. (8),

$$
\begin{aligned}
\lim _{\alpha \rightarrow \infty} & \left(\Phi_{n}^{\prime} \Omega_{n}, \frac{1}{2} \sum_{\mu=-2}^{2} G_{-\mu}\left(\mathbf{q}_{\alpha}\right) T_{\mu} \Phi_{m} \Omega_{m}\right) \\
\quad & \lim _{\alpha \rightarrow \infty} \sum_{\mu=-2}^{2}\left[\frac{1}{2} \int \overline{\Phi_{n}^{\prime}(\mathbf{x})} \Phi_{m}(\mathbf{x}) G_{-\mu}\left(\mathbf{q}_{\alpha}\right)(\mathbf{x}) d \mathbf{x}\right]\left(\Phi_{n}, T_{\mu} \Omega_{m}\right) .
\end{aligned}
$$

Again $\mathbf{q}_{\alpha} \rightarrow \mathbf{q}$ implies $G_{\mu}\left(\mathbf{q}_{\alpha}\right) \rightarrow G_{\mu}(\mathbf{q})$ as distributions, and from Eq. (7) we find that

$$
\begin{aligned}
& G_{0}(\mathbf{q})=0, \\
& G_{1}(\mathbf{q})=-\overline{G_{-1}(\mathbf{q})}=-\left(x^{3} / 2\right)\left[\left(x^{2}-i x^{1}\right) / R\right] \delta(|\mathbf{x}|-R), \\
& G_{2}(\mathbf{q})=\overline{G_{-2}(\mathbf{q})}=-\frac{1}{2}\left[\left(2 x^{1} x^{2}-i\left(R^{2}-\left(x^{3}\right)^{2}\right)\right) / R\right] \delta(|\mathbf{x}|-R) .
\end{aligned}
$$

Thus Eq. (13) consists only of surface terms, which cannot however cancel the surface term from Eq. (12). Again, the surface terms vanish when $\Phi_{m}$ vanishes on the boundary $|\mathbf{x}|=R$. Thus we see that this condition characterizes the domain $D_{0}$, and that on this domain,

$$
\lim _{\alpha \rightarrow \infty} J\left(\mathbf{q}_{\alpha}\right) \Psi=\left(J_{0}(\mathbf{q}) \cdot I+\mathbf{v} \cdot \mathbf{\Sigma}\right) \Psi \text {. Q.E.D. }
$$

It is interesting to observe why, when the limit $\mathbf{q}_{\alpha} \rightarrow \mathbf{q}$ is taken in the above theorem, the terms $(1 / 2)\left(\operatorname{curl} \mathbf{q}_{\alpha}\right) \cdot \boldsymbol{\Sigma}$ can give a representation of $\mathrm{SU}(2)$, and not merely of $\mathrm{SO}(3)$. Consider diffeomorphism group elements $\boldsymbol{\varphi}_{s}^{\mathbf{q}_{\alpha}}$ corresponding to flows under $\mathbf{q}_{\alpha}$ for fixed s. As $\mathbf{q}_{\alpha} \rightarrow \mathbf{q}$, the elements $\boldsymbol{\varphi}_{s}^{\mathbf{q}_{\alpha}}$ approximate pointwise, for $|\mathbf{x}| \neq R$, a local rigid rotation $\boldsymbol{\varphi}=\boldsymbol{\varphi}_{s}^{\mathbf{q}}$. Let $\mathbf{x}_{t}$ define a path from infinity to $\mathbf{x}$ for any fixed $\mathbf{x}$ inside the region of nontrivial $\boldsymbol{\varphi}$. Then the path in $\operatorname{SL}(3, \mathbb{R})$ given by $t \rightarrow h_{\mathbf{x}_{t}}\left(\boldsymbol{\varphi}_{s}^{\mathbf{q}_{\alpha}}\right)$ defines the element of $\overline{\mathrm{SL}}(3, \mathbb{R})$ associated with the point $\mathbf{x}$ which would be used in Eq. (4) to obtain $\mathscr{V}\left(\boldsymbol{\varphi}_{s}^{\mathbf{q}_{\alpha}}\right)$. But when the limit $\alpha \rightarrow \infty$ is taken, $t \rightarrow h_{\mathbf{x}_{t}}\left(\boldsymbol{\varphi}_{s}^{\mathbf{q}_{\alpha}}\right)$ becomes a discontinuous path in $\operatorname{SL}(3, \mathbb{R})$. Thus the choice of approximating sequence $\boldsymbol{\varphi}_{s}^{\mathbf{q}_{\alpha}}$ can affect the decision as to which element of $\overline{\mathrm{SL}(3, \mathbb{R})}$ will be associated with a point $\mathbf{x}$ inside the sphere. That is, $\lim _{\alpha \rightarrow \infty} \tilde{h}_{\mathbf{x}}\left(\boldsymbol{\varphi}_{s}^{\mathbf{q}_{\alpha}}\right)$ depends on the choice of sequence $\mathbf{q}_{\alpha}$. For a particular local rigid rotation generated by the discontinuous vector field $\mathbf{q}$, there will be two distinct equivalence classes of approximating sequences of diffeomorphisms $\boldsymbol{\varphi}_{\alpha}$, corresponding to the two classes of paths in SL $(3, \mathbb{R})$ from the identity to $\left(\operatorname{det} \mathscr{J}_{\varphi}(\mathbf{x})\right)^{-1 / 3} \mathscr{J}_{\varphi}(\mathbf{x})$ in $\operatorname{SO}(3)$. If one of these classes is exemplified by $\boldsymbol{\varphi}_{s}^{\mathbf{q}_{\alpha}}$, then the other is exemplified by $\boldsymbol{\varphi}_{s+2 \pi}^{\mathbf{q}_{\alpha}}$, where $\mathbf{q}_{\alpha} \rightarrow \mathbf{q}$ and $\boldsymbol{\varphi}_{s}^{\mathbf{q}}=\boldsymbol{\varphi}_{s+2 \pi^{\mathbf{q}}}$. Thus in some representations of $\overline{\mathrm{SL}(3, \mathbb{R})}$, the limit $\mathbf{q}_{\alpha} \rightarrow \mathbf{q}$ determines a double-valued representation of the local rigid rotation group $\mathrm{SO}(3)$, depending on which approximating sequence is used; this is just an ordinary representation of $\mathrm{SU}(2)$.

The self-adjoint operators $J(\mathbf{q})=J_{0}(\mathbf{q}) \cdot I+\mathbf{v} \cdot \mathbf{\Sigma}$ of the preceding theorem preserve the $\mathrm{SU}(2)$-invariant subspaces in $L^{2}\left(\mathbb{R}^{3}, \mathscr{M}\right)$, and generate unitary representations of groups of local rigid rotations which likewise preserve these 
subspaces. The operators $\varrho(f)$ also leave such subspaces invariant. Letting $\chi$ be the characteristic function of a bounded region, $f_{\alpha} \in \mathscr{S}$, and $\lim _{\alpha \rightarrow \infty} f_{\alpha}=\chi$ as distributions, we obtain operators $\varrho(\chi)$ corresponding to measurements of mass in the region. We call the operators $J(\mathbf{q})$ and $\varrho(\chi)$ "local measurement operators."

To summarize, one begins with a representation of the current algebra of Eq. (1) induced by a representation of $\overline{\mathrm{SL}(3, \mathbb{R})}$. One then constructs operators corresponding to measurements of angular momentum in a region invariant under rotations. Each such operator can be approximated by operators in the original current algebra on a dense domain of particle states in which the particle is localized away from the region's boundary. The algebra of these operators then leaves invariant the subspaces of the Hilbert space corresponding to representations of SU(2). Likewise, measurements of mass in a bounded region preserve these subspaces. In a theory where such local measurement operators are taken to correspond to a complete set of observables, the particle spin cannot be changed by any realizable experiment.

Next, we observe that within each SU(2)-invariant subspace of the original representation, we have a new representation of the algebra of Eq. (1), augmented by spin density operators $[1,4]$, and a corresponding representation of the local current group associated with this algebra [3]. We rewrite Eq. (1), with $J_{0}$ replacing $J$, and specify the following additional commutators:

$$
\begin{aligned}
{[\varrho(f), \Sigma(\mathbf{h})] } & =0, \\
{\left[\Sigma\left(\mathbf{h}_{1}\right), \Sigma\left(\mathbf{h}_{2}\right)\right] } & =i \Sigma\left(\mathbf{h}_{1} \times \mathbf{h}_{2}\right), \\
{\left[\Sigma(\mathbf{h}), J_{0}(\mathbf{g})\right] } & =i \Sigma(\mathbf{g} \cdot \nabla \mathbf{h}) .
\end{aligned}
$$

Of course $J_{0}(\mathbf{g})$ is to be interpreted as the kinetic (orbital) momentum density, averaged with the vector field $\mathbf{g}$, and $\Sigma(\mathbf{h})$ as the averaged spin density. The group obtained by exponentiating these commutators can be written $(\mathscr{S} \otimes \mathscr{T}) \wedge \mathscr{K}$; where $\mathscr{T}$ is the group of $C^{\infty}, \mathrm{SU}(2)$-valued functions on $\mathbb{R}^{3}$ which tend rapidly toward the identity at infinity and the group operation is pointwise multiplication, and where $\otimes$ denotes the direct product and $\wedge$ a semidirect product. For $T_{1}, T_{2} \in \mathscr{T}$, the group law for $(\mathscr{S} \otimes \mathscr{T}) \wedge \mathscr{K}$ becomes

$$
\left(f_{1}, T_{1}, \boldsymbol{\varphi}_{1}\right)\left(f_{2}, T_{2}, \boldsymbol{\varphi}_{2}\right)=\left(f_{1}+f_{2} \circ \boldsymbol{\varphi}_{1},\left(T_{1}\right)\left(T_{2} \circ \boldsymbol{\varphi}_{1}\right), \boldsymbol{\varphi}_{1} \boldsymbol{\varphi}_{2}\right) .
$$

Letting $\mathscr{D}^{(S)}$ be the usual irreducible $(2 S+1)$-dimensional representation of $\operatorname{SU}(2)$, and $\Psi(\mathbf{x})$ a $(2 S+1)$-component spinor, we obtain the one-particle spin $S$ representation $U(f) W(T) V(\boldsymbol{\varphi})$ of $(\mathscr{S} \otimes \mathscr{T}) \wedge \mathscr{K}$ :

$$
\begin{aligned}
{[U(f) \Psi](\mathbf{x}) } & =e^{i f(\mathbf{x})} \Psi(\mathbf{x}), \\
{[W(T) \Psi](\mathbf{x}) } & =\mathscr{D}^{(S)}(T(\mathbf{x})) \Psi(\mathbf{x}), \\
{[V(\varphi) \Psi](\mathbf{x}) } & =\Psi(\varphi(\mathbf{x})) \sqrt{\operatorname{det} \mathscr{J}(\mathbf{x})}
\end{aligned}
$$

The corresponding representation of the algebra (15) is

$$
\begin{aligned}
{[\varrho(f) \Psi](\mathbf{x}) } & =f(\mathbf{x}) \Psi(\mathbf{x}), \\
{[\Sigma(\mathbf{h}) \Psi](\mathbf{x}) } & =\frac{1}{2} \mathbf{h}(\mathbf{x}) \cdot \mathscr{D}^{(S)}(\boldsymbol{\sigma}) \Psi(\mathbf{x}), \\
{\left[J_{0}(\mathbf{g}) \Psi\right](\mathbf{x}) } & =\frac{1}{2 i}[\mathbf{g}(\mathbf{x}) \cdot \nabla+\nabla \cdot \mathbf{g}(\mathbf{x})] \Psi(\mathbf{x}),
\end{aligned}
$$


where the Pauli spin matrices $\sigma$ satisfy $\left[\sigma_{j}, \sigma_{k}\right]=2 i \varepsilon_{j k \epsilon} \sigma_{\ell}$. The relationship between $\Sigma(\mathbf{h})$ and $W(T)$ may be described as follows: let $T_{\mathbf{h}}(\mathbf{x})=\exp \left[\frac{1}{2} i \mathbf{h}(\mathbf{x}) \cdot \boldsymbol{\sigma}\right]$; then $T_{\mathbf{h}} \in \mathscr{T}$ and $W\left(T_{\mathbf{h}}\right)=\exp [i \Sigma(\mathbf{h})]$. We observe that if $R(\hat{n}, \theta) \mathbf{y}$ denotes the vector obtained by rotating the vector $\mathbf{y}$ about the unit vector $\hat{n}$ by the angle $\theta$, then we have $\exp \left[i \Sigma\left(\mathbf{h}_{1}\right)\right] \exp \left[i \Sigma\left(\mathbf{h}_{2}\right)\right] \exp \left[-i \Sigma\left(\mathbf{h}_{1}\right)\right]=\exp \left[i \Sigma\left(R\left(\hat{h}_{1},-\left|\mathbf{h}_{1}\right|\right) \mathbf{h}_{2}\right)\right][20]$.

Finally, we remark that the representation of Eq. (18) can be obtained in a straightforward manner from the local measurement operators $\varrho(\chi)$ and $J(\mathbf{q})$ constructed earlier, together with the total momentum operator $\mathbf{P}=\int \mathbf{J}(\mathbf{x}) d \mathbf{x}$. The operators $\varrho(f)$ are first recovered: approximating $f$ by finite linear combinations of characteristic functions $\chi_{j}$, one takes a limit of linear combinations of $\varrho\left(\chi_{j}\right)$. Next, $J_{0}(\mathbf{g})$ can be written $\frac{1}{2} \sum_{k=1}^{3}\left[\varrho\left(g^{k}\right) P_{k}+P_{k} \varrho\left(g^{k}\right)\right]$, where $P_{k}$ is the $k^{\text {th }}$ component of $\mathbf{P}$. As before, suppose that $\mathbf{q}_{\alpha}$ is a sequence of $C^{\infty}$ vector fields tending toward $\mathbf{q}$; the local orbital angular momentum operator $J_{0}(\mathbf{q})$ can now be constructed on an appropriate domain as a limit of operators $J_{0}\left(\mathbf{q}_{\alpha}\right)$. Now $\mathbf{v} \cdot \mathbf{\Sigma}=J(\mathbf{q})-J_{0}(\mathbf{q})$. Finally $\Sigma(\mathbf{h})$ is obtained by approximating $\mathbf{h}$ with linear combinations of characteristic vector fields $\mathbf{v}_{j}$, and taking a limit of linear combinations of $\mathbf{v}_{j} \cdot \mathbf{\Sigma}$.

Representations of $\mathscr{S} \wedge \mathscr{K}$ induced by representations of $\overline{\mathrm{SL}(3, \mathbb{R})}$ have also been given which describe $N$ particles for $N>1$ [13]. Consider for example an $N$-tuple of distinct representations $\left(\pi_{1}, \ldots, \pi_{N}\right)$ of $\left.\overline{\mathrm{SL}(3, \mathbb{R})}\right)$ In the induced representation, the operators $\mathscr{U}(f)$ and $\mathscr{V}(\boldsymbol{\varphi})$ act on the tensor product Hilbert space $\mathscr{H}=L^{2}\left(\mathbb{R}^{3}, \mathscr{M}_{1}\right) \otimes \ldots \otimes L^{2}\left(\mathbb{R}^{3}, \mathscr{M}_{N}\right)$, where $\mathscr{M}_{j}$ is the representation space of $\pi_{j}$. The representation is given by linearly extending the operators

$$
\begin{gathered}
{\left[\mathscr{U}(f) \bigotimes_{j=1}^{N} \Psi_{j}\right]\left(\mathbf{x}_{1}, \ldots, \mathbf{x}_{N}\right)=\bigotimes_{j=1}^{N} e^{i f\left(\mathbf{x}_{j}\right)} \Psi_{j}\left(\mathbf{x}_{j}\right),} \\
{\left[\mathscr{V}(\boldsymbol{\varphi}) \bigotimes_{j=1}^{N} \Psi_{j}\right]\left(\mathbf{x}_{1}, \ldots, \mathbf{x}_{N}\right)=\bigotimes_{j=1}^{N} \pi_{j}\left(\tilde{h}_{\mathbf{x}_{j}}(\boldsymbol{\varphi})\right) \Psi_{j}\left(\boldsymbol{\varphi}\left(\mathbf{x}_{j}\right)\right) \sqrt{\operatorname{det} \mathscr{J}_{\boldsymbol{\varphi}}\left(\mathbf{x}_{j}\right)},}
\end{gathered}
$$

generalizing Eqs. (4) and (5). The local currents may be written

$$
\begin{aligned}
\varrho(f)= & \sum_{j=1}^{N} f\left(\mathbf{x}_{j}\right) \cdot I, \\
J(\mathbf{g})= & \sum_{j=1}^{N} \frac{1}{2 i}\left[\mathbf{g}\left(\mathbf{x}_{j}\right) \cdot \nabla_{j}+\nabla_{j} \cdot \mathbf{g}\left(\mathbf{x}_{j}\right)\right] \cdot I+\sum_{j=1}^{N} \frac{1}{2}\left(\operatorname{curl} \mathbf{g}\left(\mathbf{x}_{j}\right) \cdot \pi_{j}(\mathbf{\Sigma})\right. \\
& +\sum_{j=1}^{N} \frac{1}{2} \sum_{\mu=-2}^{2} G_{-\mu}(\mathbf{g})\left(\mathbf{x}_{j}\right) \cdot \pi_{j}\left(T_{\mu}\right),
\end{aligned}
$$

where each term has its obvious interpretation as an operator in the tensor product space $\mathscr{H}$.

Now one can construct the local measurement operators as in the one-particle case above. Invariant subspaces for local measurement operators are tensor products of SU(2)-invariant subspaces - i.e. $\mathscr{W}=\bigotimes_{j=1}^{N} \mathscr{W}_{j}$, where $\mathscr{W}_{j}=L^{2}\left(\mathbb{R}^{3}, \mathscr{N}_{j}\right)$ $C L^{2}\left(\mathbb{R}^{3}, \mathscr{M}_{j}\right)$, and $\mathscr{N}_{j}$ is a subspace of $\mathscr{M}_{j}$ invariant for an irreducible representation of $\mathrm{SU}(2)$. If the representations of $\mathrm{SU}(2)$ for some subset of the spaces $\mathscr{N}_{j}$ are unitarily equivalent, then $\mathscr{W}$ decomposes further into a direct sum of 
subspaces satisfying various exchange symmetry conditions. Thus, though the particles are initially regarded as distinct because the representations $\pi_{j}$ are distinct, the absence of local measurement operators connecting the different $\mathrm{SU}(2)$-invariant subspaces in $L^{2}\left(\mathbb{R}^{3}, \mathscr{M}_{\mathrm{j}}\right)$ means that vectors in $\mathscr{W}$ now describe some particles which cannot be distinguished, and which may satisfy for example Bose or Fermi statistics. We believe that in $N$-particle representations a proof can be given that local measurement operators form a complete set of observables, but the elementary argument given above does not generalize to the case of more than one particle.

\section{Discussion}

To sum up, we start with a continuous unitary representation of $\mathscr{S} \wedge \mathscr{K}$, from which we obtain the current algebra of self-adjoint operators which generate oneparameter unitary subgroups. We consider the operators obtained when the test functions indexing the local currents approach (in the case of $\varrho$ ) characteristic functions, or (in the case of $J$ ) vector fields generating local rigid rotations. These choices for test functions correspond to measurements of particle number in a region, or of angular momentum in a rotation-invariant region. The resulting "local measurement operators" are defined on dense domains of wave functions vanishing at the regions' boundaries. Such wave functions describe the physical states on which the measurements can be performed.

When the original representation of $\mathscr{S} \wedge \mathscr{K}$ is induced by a representation $\pi$ of $\overline{\mathrm{SL}(3, \mathbb{R})}$, or more generally by representations $\pi_{1}, \ldots, \pi_{N}$ of $\overline{\mathrm{SL}(3, \mathbb{R})}$, the Hilbert space decomposes into a direct sum of subspaces invariant for the local measurement operators. Such subspaces are (tensor products of) SU(2)-invariant subspaces, and may be interpreted as describing particles with spin. In each such subspace, a representation of the local current group $(\mathscr{S} \otimes \mathscr{T}) \wedge \mathscr{K}$ and its generators can be constructed in terms of the local measurement operators. Thus, the description of particles with spin in which the local SU(2) current group is adjoined "by hand" to the group $\mathscr{S} \wedge \mathscr{K}$, the latter being generated by the mass and the (orbital) momentum densities, is obtained from a unified description in which the group $\mathscr{S} \wedge \mathscr{K}$ is generated by the mass and the total momentum densities. We also remark that if this procedure is followed for representations of $\mathscr{S} \wedge \mathscr{K}$ without spin, one obtains local measurement operators for which there are no nontrivial proper invariant subspaces of the Hilbert space. The original (spin zero) representation of $\mathscr{S} \wedge \mathscr{K}$ can in this case be reconstructed from local measurement operators.

Let us discuss further the interpretation that only the above-mentioned classes of test functions give operators corresponding to physical measurements. To interpret a measurement of $\varrho(f)$, where $f \in \mathscr{S}$, imagine an apparatus with the capability of giving a "yes or no" answer to the question of whether a particle is located in any particular region of very small volume. This allows measurement of the expectation value of $\varrho(\chi)$, where $\chi$ is the characteristic function of such a region. Now one can approximate the expectation value of $\varrho(f)$ by averaging the results of small-volume measurements with appropriate weights. Equivalently, one can imagine a particle detection apparatus where $f(\mathbf{x})$ describes the efficiency of the 
detector in a small region about $\mathbf{x}$. Thus, in the representations discussed in this paper, the $\varrho(\chi)$ can always be recovered from the $\varrho(f)$, and vice versa.

The situation for the operators $J(\mathrm{~g})$ is not so simple. One would like to think of measuring the linear momentum in small regions and taking an average, but we have noted that there are no self-adjoint operators corresponding to measurements of linear momentum in bounded regions. There do exist self-adjoint operators for measurements of angular momentum in bounded regions with rotation symmetry about the direction of the measured angular momentum component. The local rigid rotation generators $J(\mathbf{q})=J_{0}(\mathbf{q}) \cdot I+\mathbf{v} \cdot \mathbf{\Sigma}$ are bona fide self-adjoint operators corresponding to measurements of total (orbital plus spin) angular momentum in such regions. The orbital momentum density operators $J_{0}(\mathbf{g})$ can be defined in the one-particle case in terms of mass densities and the total momentum. This permits the orbital angular momentum $J_{0}(\mathbf{q})$ to be obtained separately, and now the spin density $\Sigma(\mathbf{h})$ for $h^{k} \in \mathscr{S}$ can be expressed as an average over small-volume measurements.

Thus, in the representation of the Lie algebra of operators $\varrho(f), J_{0}(\mathbf{g})$, and $\Sigma(\mathbf{h})$, which we finally obtain on each SU(2)-invariant subspace, each test function can be considered as a weighting function averaging measurements made in small regions. But measurements such as these do not change the particle spin. How then are we to understand the terms $\frac{1}{2} \sum_{\mu=-2}^{2} G_{-\mu}(\mathbf{g}) T_{\mu}$ in the expression for $J(\mathbf{g})$, which cannot be expressed as averages of local mass and angular momentum measurements? These terms are non-vanishing when $\mathbf{g}$ is the generator of diffeomorphisms which "dilate" space non-uniformly in some region. Let $\varphi_{t}^{\mathbf{g}}$ be the one-parameter group generated by such a dilation. In the representation $V\left(\boldsymbol{\varphi}_{t}^{\mathbf{g}}\right)$ $=\exp \left[i t J_{0}(\mathbf{g})\right]$ which acts only on spatial coordinates, we have

$$
\left[V\left(\varphi_{t}^{\mathrm{g}}\right) \Psi\right](\mathbf{x})=\Psi\left(\boldsymbol{\varphi}_{t}^{\mathrm{g}}(\mathbf{x})\right) \sqrt{\operatorname{det} \mathscr{J}_{\varphi_{t}^{\mathrm{g}}(x)}} .
$$

Here $V\left(\boldsymbol{\varphi}_{t}^{\mathbf{g}}\right)$ can be thought of not as implementing an actual spatial deformation, but simply as repositioning the particles in (undeformed) space. These two notions can be treated as indistinguishable for non-relativistic point particles having no spin degrees of freedom. The interpretation of $V\left(\varphi_{t}^{\mathbf{g}}\right)$ as a repositioning parallels the interpretation of $J_{0}(\mathbf{g})$ as a weighted average of the momentum operators $\mathbf{P}$.

However, if the particles have internal structure or spin, then repositioning them in space is not the same thing as actually dilating space, since the latter would affect the internal structure while the former would not. In the representation $\mathscr{V}\left(\varphi_{t}^{\mathbf{g}}\right)=\exp [i t J(\mathbf{g})]$, we may think of $J(\mathbf{g})$ as the generator of an actual spatial dilation, which thus connects the different spin subspaces. One might, for example, think of dilations of space such as would occur in the presence of a strong gravitational field, and conjecture that the ability to produce and manipulate such a field in the laboratory could produce superpositions of states describing a point particle with different spins $[21,22]$. In the absence of such fields, however, $J(\mathbf{g})$ must be interpreted as a physical measurement operator only as $\mathbf{g}$ approaches a local rigid rotation generator. Its domain of definition in this limit must be states in which particles are not present at boundaries where the dilation is nonvanishing as the limit is taken. This is the procedure that we have adopted in the present paper. 
Acknowledgements. We are indebted to R. Menikoff for stimulating discussions. G. G. thanks Los Alamos National Laboratory and D. H. Sharp thanks Princeton University for hospitality. Financial support was provided by the U.S. Department of Energy.

\section{References}

1. Dashen, R.F., Sharp, D.H.: Currents as coordinates for hadrons. Phys. Rev. 165, 1857 (1968)

2. Goldin, G.A., Sharp, D.H.: Lie algebras of local currents and their representations. In: Group Representations in Mathematics and Physics, Battelle Seattle 1969 Rencontres. Bargmann, V. (ed.), pp. 300-311. New York: Springer 1970

3. Goldin, G.A. : Nonrelativistic current algebras as unitary representations of groups. J. Math. Phys. 12, $462(1971)$

4. Grodnik, J., Sharp, D.H.: Description of spin and statistics in nonrelativistic quantum theories based on local currents. Phys. Rev. D 1, 1546 (1970)

5. Araki, H.: Factorizable representation of current algebra. Vol. 5, pp. 361-422. Publ. RIMS, Kyoto University 1969/70

6. Streater, R.: Current commutation relations, continuous tensor products and infinitely divisible group representations. In: Local quantum theory. Jost, R. (ed.), pp. 247-263. New York: Academic 1969

7. Ismagilov, R.S.: Mat. Sbornik 100, 117 (1976)

8. Vershik, A.M., Gel'fand, I.M., Graev, M.I.: Dokl. Akad. Nauk. SSSR. 232, 745 (1977)

9. Guichardet, A.: Représentations de $G^{X}$ ( $G$ compact) selon Verchik-Gelfand-Graiev et Ismagilov. Séminaire Bourbaki Vol. 1978/79, Exposés 525-542. In: Lecture notes in mathematics, Vol. 770, pp. 303-311. New York: Springer 1980

10. Goldin, G.A., Menikoff, R., Sharp, D.H.: Particle statistics from induced representations of a local current group. J. Math. Phys. 21, 650 (1980)

11. Borisov, A.B.: The unitary representations of the general covariant group algebra. J. Phys. A: Math. Gen. 11, 1057 (1978)

12. Borisov, A.B.: The unitary representations of the Diff $R^{N}$ group. J. Phys. A: Math. Gen. 12, 1625 (1979)

13. Goldin, G.A., Menikoff, R., Sharp, D.H.: J. Phys. A: Math. Gen. 16, 1827 (1983)

14. Goldin, G.A., Grodnik, J., Powers, R.T., Sharp, D.H.: Nonrelativistic current algebra in the $N / V$ limit. J. Math. Phys. 15, 88 (1974)

15. Goldin, G.A., Menikoff, R., Sharp, D.H.: Induced representations of diffeomorphism groups described by cylindrical measures. In: Measure theory and its applications. Goldin, G.A., Wheeler, R.F. (eds.), pp. 207-218. Dept. of Mathematical Sciences, Northern Illinois University, DeKalb, Ill, 1981

16. Goldin, G.A., Sharp, D.H.: Rotation generators in two-dimensional space and particles obeying unusual statistics. Phys. Rev. Ser. D28, 830 (1983)

17. Goldin, G.A., Menikoff, R. : Quantum-mechanical representations of the group of diffeomorphisms and local current algebra describing tightly bound composite systems. Los Alamos preprint (1983)

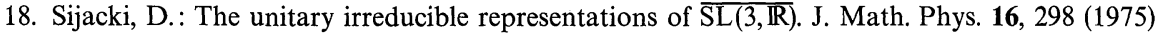

19. Weaver, L., Biedenharn, L.C., Cusson, R.Y.: Rotational bands in nuclei as induced group representations. Ann. Phys. 77, 250 (1973)

20. Sign errors in Ref. [3], Eqs. (3.38) and (3.39), are corrected here

21. Friedman, J.L., Sorkin, R.D.: Spin $\frac{1}{2}$ from gravity. Phys. Rev. Lett. 44, 1100 (1980)

22. Isham, C.J.: Quantum geometry (1982 preprint, to appear in the Festschrift in honor of Bryce DeWitt's sixtieth birthday; published by Adam Hilger)

Communicated by R. Haag

Received June 20, 1983 